\title{
Mehragrarimporte aus Entwickungsländern ; Möglichkeiten und Grenzen
}

Rudolf Horber

\section{(2) OpenEdition \\ 1 Journals}

Electronic version

URL: http://journals.openedition.org/sjep/1177

DOI: $10.4000 /$ sjep. 1177

ISSN: 1663-9677

Publisher

Institut de hautes études internationales et du développement

Printed version

Date of publication: 1 janvier 1988

Number of pages: 187-202

ISSN: 1660-5926

\section{Electronic reference}

Rudolf Horber, « Mehragrarimporte aus Entwickungsländern ; Möglichkeiten und Grenzen »,

Schweizerisches Jahrbuch für Entwicklungspolitik [Online], 7 | 1988, Online erschienen am: 05 April 2013, abgerufen am 08 September 2020. URL : http://journals.openedition.org/sjep/1177 ; DOI : https:// doi.org/10.4000/sjep. 1177 


\title{
Mehragrarimporte aus Entwickungsländern; Möglichkeiten und Grenzen
}

\author{
RudolfHorber
}

\section{Résumé:}

Environ $20 \%$ de nos importations en produits agricoles proviennent des pays en voie de développement. Nous savons que ces pays sont eux-mémes fortement tributaires de lexportation de produits agncoles, ne serait-l dono pas possible d'améliorer en leur faveur laccés au marché suisse ainsi qu aux autres marchés des pays développés? Cette question s'impose d'autant plus que les PVD offrent de grands avantages comparatifs au niveau des matieres premieres.

Dans cet article, lauteur tente a démontrer, en stappuyant sur différents criter res. qu un en couragement approprié des importations agricoles en provenance des PVD n'a de sens que si certaines conditions dans ces pays mémes sont remplies. Mais quoi qúil en soit, des mesures autonomes et isolées prises par la suisse dans le but de favoríser les importations des produits agricoles en provenance des PVD ne sauraient étre suffisantes. Il est encore plus important de renforcer les mesures prises dans ces pays afin de soutenir davantage et durablement le secteur agricole, et de stimuler les elforts déployés collectivement par les pays industrialisés dans le but dassainir les marches mondiaux en réduisant leur production exédentairo et; subséquemment, en améliorant les possibilités dexportation des PVD. 


\section{Einleitung und Problemstellung}

Die Entwicklungsländer sind nach der Europäischen Gemeinschaft unser wichtigster Agrarhandelspartner, und verschiedene Drittweltstaaten sind auf den Export von Landwirtschaftsprodukten essentiell angewiesen. Trotzdem hat unser Agrarhandel mit den Entwicklungsländern bisher weder in der theoretischen noch in der praxisbezogenen Literatur grössere Beachtung gefunden. Dies hängt vielleicht mit der Tatsache zusammen, dass die Frage, ob mit den Drittweltstaaten mehr oder weniger (Agrar)-Handel zu treiben sei, in der Theorie wie auch in der Praxis ziemlich umstritten ist.

Ausgehend von einer Bestandsaufnahme der schweizerischen Agrarhandelsbeziehungen mit den Entwicklungsländern soll daher in diesem Beitrag anhand verschiedener Kriterien untersucht werden, unter welchen Voraussetzungen sich Mehrbezüge von Landwirtschaftsgütern aus den Drittweltstaaten überhaupt aufdrängen. Im einzelnen beginnt der Artikel mit einer Darstellung des Agrarhandels mit den Entwicklungsländern nach Produkten und Ländern, wobei anhand einiger Beispiele auch auf die Bedeutung der Landwirtschaftsexporte für die Dritte Welt hingewiesen wird. In einem nächsten Kapitel wird die schweizerische Agrarhandelspolitik gegenüber den Entwicklungsländern bezüglich Zielsetzung und Massnahmen dargelegt. Das zentrale vierte Kapitel versucht die heikle Frage "Mehr Agrarimporte aus Drittweltstaaten?" unter Einbezug entwicklungs-, agrarsowie wirtschafts- und ordnungspolitischer Überlegungen zu beantworten und gleichzeitig aufzuzeigen, welche Veränderungen auf nationaler und multilateraler Ebene vorgenommen werden müssen, um die Exportmöglichkeiten der Entwicklungsländer zu verbessern.

Eingangs sind noch zwei häufig verwendete Schlüsselbegriffe zu definieren. Aus praktisch-statistischen Gründen wird der Kreis der Entwicklungsländer wie folgt gezogen: alle asiatischen Staaten ausser den kommunistischen Ländern und Japan; sämtliche Staaten Afrikas ohne die Südafrikanische Republik; alle Länder Amerikas mit Ausnahme der USA und Kanadas; Jugoslawien, Gibraltar, Zypern und Malta als europäische Staaten. Zur Umschreibung des Agrarhandels erweisen sich eine weitere und eine engere Definition als zweckmässig. Erstere soll die Waren der Kapitel 1-24 des Zolltarifs, die auch landwirtschaftliche Verarbeitungsprodukte höherer Stufen einschliessen, umfassen, während sich letztere auf die sogenannten Basisagrarprodukte gemäss EG-Definition beschränkt.

\section{Entwickung des Agrarhandels mit den Drittweltstaaten}

\section{1. Überblick}

Die Entwicklungs/änder sind nach wie vor stark von Rohstoffexporten abhängig. Dabei kommt den Landwirtschaftsprodukten verschiedentlich ein hoher Stellenwert zu. So entfallen nach Angaben der FAO gegen die Hälfte der Exporterlöse der erdölimportierenden Länder Afrikas und Lateinamerikas auf Agrargüter; für 
sämtliche Drittweltstaaten waren es 1984 immerhin 17 Prozent, was einem Ausfuhrwert von 88 Milliarden US-Dollar entspricht. Für verschiedene Entwicklungsländer ist diese Agrarexportquote aber deutlich über 50 Prozent, wie die folgenden Beispiele für 1984 aufzeigen:

Tabelle Nr. 1

\section{Agrarexportlastigkelt elnzelner Drittweltstaaten}

\begin{tabular}{|c|c|c|c|}
\hline Land & $\begin{array}{l}\text { Gesamtausfuhren } \\
\text { (Mio US-\$) }\end{array}$ & $\begin{array}{c}\text { Agrarausfuhren } \\
\text { (Mio US } \$ \text { ) }\end{array}$ & Agrarexportquote \\
\hline Tansania & 456 & 389 & 85 \\
\hline Paraguay & 381 & 303 & 80 \\
\hline Honduras & 746 & 527 & 71 \\
\hline Kenia & $1^{\prime} 078$ & 747 & 69 \\
\hline Elfenbeinküste & 2703 & $1 ' 858$ & 69 \\
\hline Ghana & 571 & 388 & 68 \\
\hline Kolumbien & 3,483 & $2 ' 364$ & 68 \\
\hline Sri Lanka & 1.454 & 897 & 62 \\
\hline Guatemala & $1 ' 129$ & 691 & 61 \\
\hline Thailand & 7413 & $3^{\prime} 821$ & 52 \\
\hline
\end{tabular}

Quelle: Weltbankbericht 1986; FAO Trado Yearbook 1985.

Aber auch für die Schweiz ist der landwirtschaftliche Warenaustausch mit den Drittweltstaaten von grosser Bedeutung: Die Entwicklungsländer sind nach der Europäischen Gemeinschaft unser zweitwichtigster Agrarhandelspartner. Wie aus Abbildung 1 hervorgeht, stammten im Mittel der Jahre 1983/8521 Prozent unserer landwirtschaftlichen Importe im Werte von 1,25 Milliarden Franken aus der Dritten Welt, und 17 Prozent unserer Agrarexporte (0,35 Milliarden Franken) fanden dort ihren Absatz. Entsprechend ist auch ein negativer Saldo im landwirtschaftlichen Warenaustausch mit den Entwicklungsländern zu verzeichnen (0,9 Milliarden 1983/85), ganz im Gegensatz zum übrigen Handel mit den Drittweltstaaten, wo beispielswiese im Jahre 1985 Exporten von 12,0 Milliarden Franken Einfuhren von lediglich 5,2 Milliarden Franken gegenüberstanden. Zu beachten ist allerdings, dass die Länderangaben der Aussenhandelsstatistik nicht immer dem tatsächlichen Ursprungs- oder Bestimmungsland entsprechen; so dürtten besonders Drittweltprodukte zum Teil den (statistischen) Umweg über die EG nehmen, bevor sie als "EG-Erzeugnisse" in die Schweiz gelangen. 


\section{Abbildung 1: Agrarhandel der Schweiz nach Ländergruppen 1)}
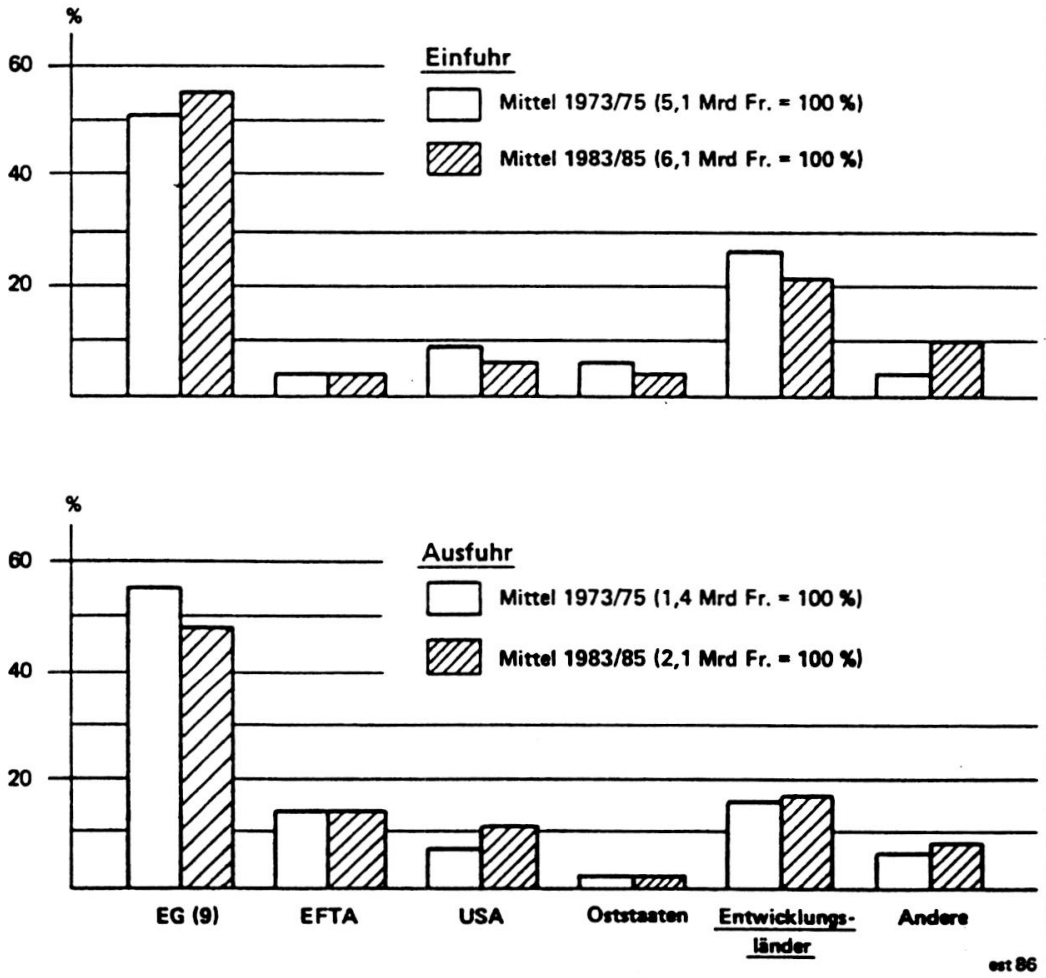

Qvelle: Bundeamt für Auseenwirtechaft, Commerce extërieur epricole suises, Ausgebe 1836.

1) Nech Kepitel 1-24 des Zollterife.

Stellenwert und Entwicklung des Agrarhandels mit den Entwicklungsländern im einzelnen gehen aus Tabelle 2 hervor. Ein Blick auf diese Zusammenstellung zeigt deutlich, dass der Agrarimportanteil aus den Drittweltstaaten tendenziell rückläufig ist, wobei sich in den letzten Jahren das Blatt allerdings wieder leicht zugunsten der Entwicklungsländer gewendet hat. Bei mengenmässiger Betrachtung ergibt sich übrigens ein ähnliches Bild: Von 1973/75 bis 1983/85 sind die Importe um über 100.000 Tonnen oder gegen 20 Prozent zurückgegangen. Dieser Marktanteilverlust der Entwicklungsländer ist neben den zum Teil unzureichenden Liefermöglichkeiten vor allem auf die mit Hilfe von Ausfuhrsubventionen geförderten Agrarexporte der EG-Staaten zurückzuführen (z.B. Zucker). Es überrascht daher nicht, dass die EG-Länder ihre Stellung auf dem Schweizer Markt festigen konnten. Mit 


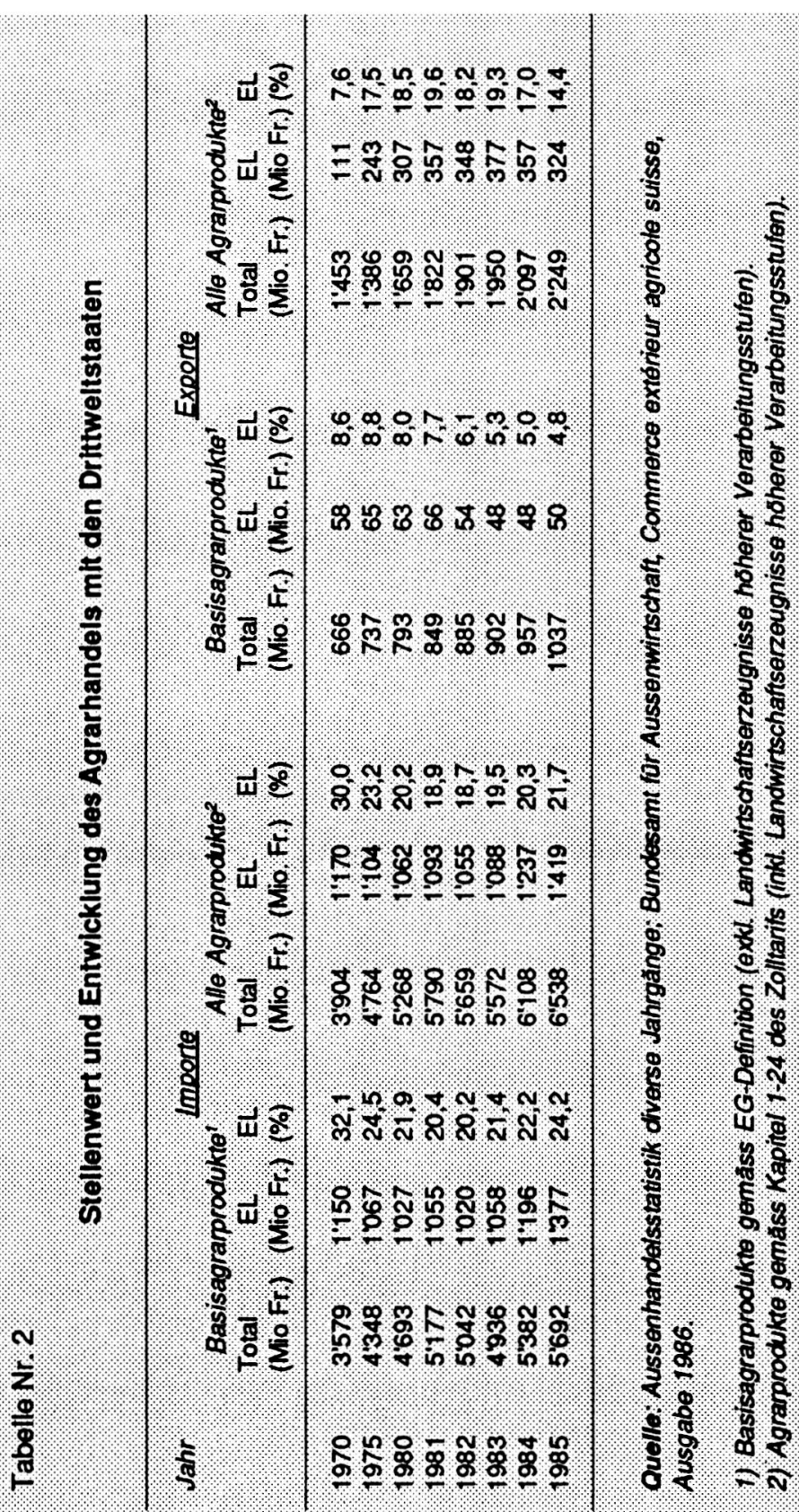


den Ausfuhren von Landwirtschaftsprodukten verhält es sich gerade umgekehrt: Rückgang des Lieferanteils in die EG und leichte Erhöhung nach den Drittweltstaaten, was in erster Linie der stärkeren Nachfrage der Ölländer nach Nahrungsmitteln zuzuschreiben ist (vgl. Abbildung Agrarhandel der Schweiz nach Länderquoten).

Die landwirtschaftlichen Einfuhren aus den Entwicklungsländern setzen sich übrigens, wie aus Tabelle 3 hervorgeht, vor allem aus Basisprodukten zusammen; auf verarbeitete Nahrungsmittel entfallen nur rund 3 Prozent. Dies kann so wenig überraschen wie die Tatsache, dass unsere Agrarexporte zu über 80 Prozent aus Verarbeitungserzeugnissen bestehen. Damit konzentrieren sich die Importe aus den Drittweltstaaten noch stärker auf Basisagrarprodukte und die Exporte dorthin ausgeprägter auf Nahrungsmittel, als dies mit den übrigen Handelspartnern der Fall ist.

\subsection{Importanalyse}

Über die wichtigsten aus den Entwicklungsländern bezogenen Agrarprodukte gibt Tabelle 3 Auskunft. Danach entfielen im Mittel der Jahre 1983/85 mehr als ein Drittel unserer wertmässigen Landwirtschaftseinfuhren aus den Drittweltstaaten auf Kaffee. An nächster Stelle folgen, allerdings mit bereits deutlichem Abstand, Rohkakao, tropische Früchte (Bananen), Fleisch, Schalenfrüchte (Mandeln und Nüsse) sowie Rohtabak. Die sogenannten Kolonialwaren sind somit nach wie vor die Hauptexportprodukte der Drittweltstaaten nach der Schweiz (und anderswohin).

Berücksichtigt wurden in Tabelle 3 jene zwölf Produktegruppen, die entweder 1973/75 oder 1983/85 einen Wert von mehr als 30 Millionen Franken erreichten. Es befinden sich keine Verarbeitungsprodukte darunter. Gegenüber der ersten Untersuchungsperiode haben namentlich Kaffee, Rohkakao, Bananen und Fruchtsäfte Boden gut gemacht, wogegen vor allem Fleisch, Rohtabak, Ölsaaten, Agrumen, Frischgemüse und Wein an Terrain verloren haben. Bei mengenmässiger Betrachtung sieht die Entwicklung für die Drittweltstaaten noch ungünstiger aus: Die Kaffeeimporte stagnieren bei etwas über 60.000 Tonnen, und von den übrigen elf untersuchten Produktegruppen haben nur gerade der Rohkakao, die Fruchtsäfte sowie die pfanzlichen Öle und Fette deutlich zugelegt, während die Bezügevon Rohtabak, Ölsaaten, Agrumen, Frischgemüse und Wein mengenmässig stark zurückgegangen sind, zum Teil bis zur Hälfte und mehr.

Untersucht man unsere Agrarimporte aus den Drittweltstaaten nach Ländern, so fällt sofort die Vorrangstellung Brasiliens auf: rund 20 Prozent unserer wertmässigen Landwirtschaftseinfuhren aus den Entwicklungsländern entfallen auf dieses lateinamerikanische Schwellenland. Wie aus Tabelle 4 hervorgeht, dominieren vor allem die lateinamerikanischen "Kaffee - und Bananenländer", sowie gewisse Mittelmeerstaaten.

Neben Brasilien haben gegenüber $1974 / 75$ vor allem Kolumbien, die Elfenbeinküste, Jugoslawien und Honduras ihre Stellung auf dem Schweizermarkt stark 


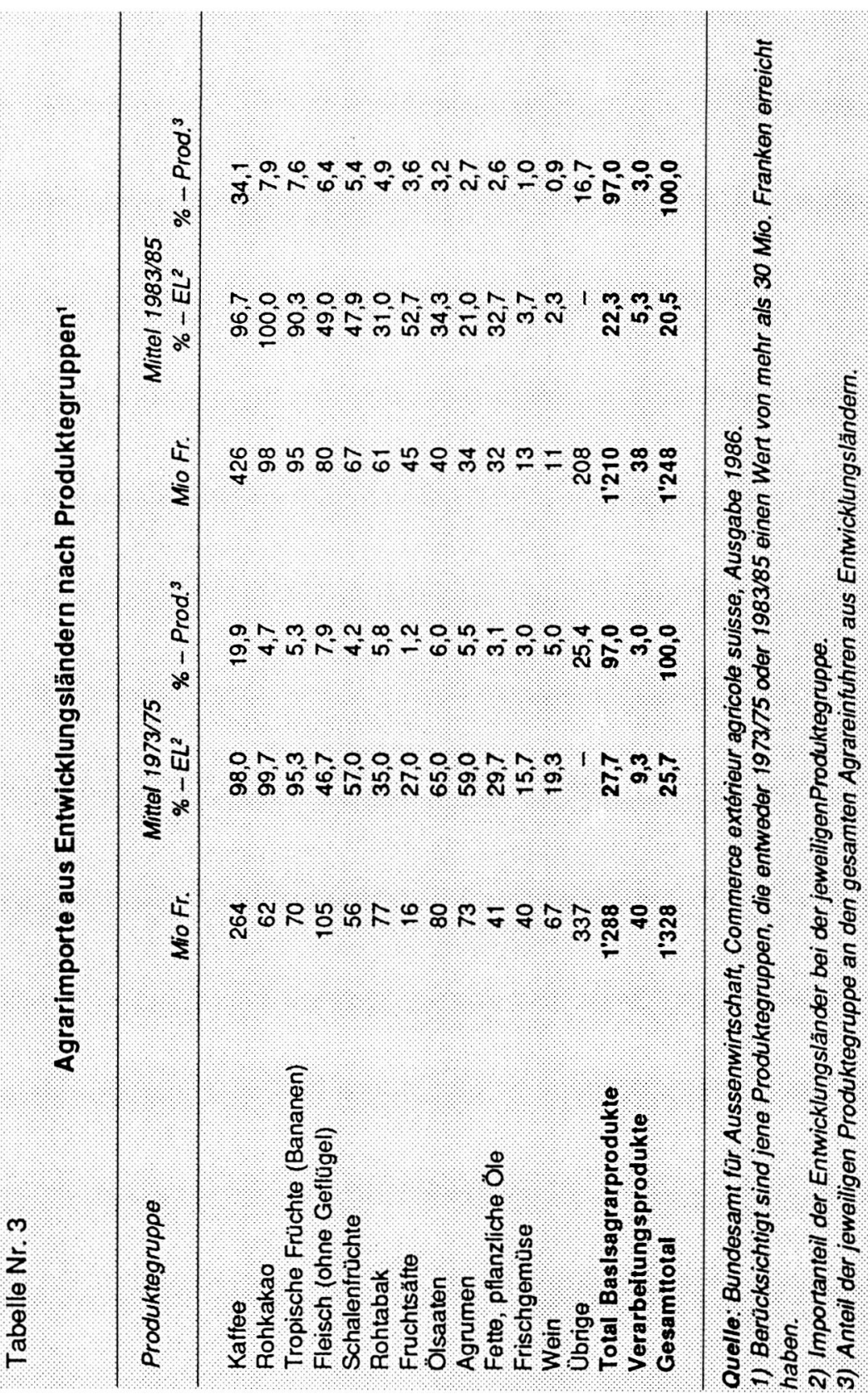


Tabelle 4

\section{Schweizerische Agrarimporte aus Drittweltstaaten nach Ländern?}

\begin{tabular}{|c|c|c|c|c|}
\hline Land & $\begin{array}{l}\text { Importo } \\
\text { (Mio. Fr.) }\end{array}$ & $\frac{197475}{(\%-A n t e i l)}$ & (mporto & $\begin{array}{l}1974 / 75 \\
(\%-A n t e i l)\end{array}$ \\
\hline Brasilien & 114 & 8,8 & 256 & 19,3 \\
\hline Argentinien & 88 & 6,8 & 94 & 7,1 \\
\hline Israel & 59 & 4,5 & 90 & 6,8 \\
\hline Kolumbien & 27 & 2,1 & 84 & 6,3 \\
\hline Turkei & 56 & 4,3 & 75 & 5,6 \\
\hline Costa Rica & 38 & 2,9 & 58 & 4,4 \\
\hline Elfenbeinkúste & 20 & 1,5 & 53 & 4,0 \\
\hline Jugoslawien & 24 & 1,8 & 48 & 3,6 \\
\hline Honduras & 13 & 1,0 & 44 & 3,3 \\
\hline Guatemala & 24 & 1,8 & 41 & 3,1 \\
\hline Ubrige & 837 & 64,5 & 485 & 36.5 \\
\hline Total & 1300 & 100 & 1328 & 100 \\
\hline
\end{tabular}

Quelle: Bundesamt for Aussenwirtschaft, Commerce exterieur suisse, Ausgabe 1986.

1) Nach Kapiteln 1-24 des Zolltarís; es sind die zehn wichtigsten Lieferlander im Mittel 198485 berucksichtigt worden.

ausbauen können; diese Länder verzeichnen innerhalb von zehn Jahren mehr als eine Verdoppelung ihrer wertmässigen Agrarexporte nach der Schweiz.

\section{Die heutige Agrarimportpolitik gegenüber den Entwicklungsländern}

\subsection{Zielsetzung und multilateraler Rahmen}

Aus nationaler Sicht ist von der generellen Zielsetzung der schweizerischen Agrarhandelspolitik auszugehen. Danach sind die agrarpolitischen Massnahmen in sinnvoller Weise zu ergänzen, um die verschiedenen Oberziele der Agrarpolitik, wie Vorsorge für Krisenzeiten, Landschaftspflege und Bewahrung der dezentralisierten Besiedlung möglichst optimal verwirklichen zu können. Konkret geht es um die Erleichterung des Absatzes der einheimischen Produktion. Dies geschieht 
einerseits durch das Bemühen, die Exportmöglichkeiten für schweizerische Agrarerzeugnisse im Ausland zu sichern und womöglich zu erweitern, anderseits aber vor allem durch das vielfältige Schutzinstrumentarium an der Grenze zur Importregulierung.

Diese generelle Zielsetzung gilt grundsätzlich auch gegenüber den Entwicklungsländern. Eine zu weitgehende Vorzugsbehandlung der Agrarimporte aus der Dritten Welt könnte die Absatzsicherung verschiedener Inlandprodukte wie gewisse Fleisch-oder Früchtearten in Frage stellen. Aus entwicklungspolitischen Gründen ist jedoch eine gewisse Bevorzugung der Agrarausfuhren aus Drittweltstaaten vorgesehen. So figuriert im Sechsten Landwirtschaftsbericht des Bundesrates unter den Einzelzielen auch die "Begünstigung der Agrarexporte der Entwicklungsländer". Dadurch sollen die Ausfuhrmöglichkeiten der Dritten Welt verbessert werden, um ihnen zu den notwendigen Deviseneinnahmen zur Entwicklung ihrer Volkswirtschaften - und zur Finanzierung des Schulden- und Zinsendienstes - zu verhelfen.

Die Rahmenbedingungen für den internationalen Agrarhandel sind gegenwärtig als ausgesprochen ungünstig zu bezeichnen. Einem nach wie vor steigenden Angebot steht eine stagnierende kaufkraftmässige Nachfrage gegenüber, und die protektionistischen und marktverzerrenden Tendenzen, wie beispielsweise die Liquidierung von Überschüssen mit Exportsubventionen nehmen weiterhin zu. Während die Nominalpreise für Agrarprodukte kürzerfristig zum Teil extremen Schwankungen ausgesetzt sind (Paradebeispiel Zucker!), verlaufen die Realpreise in der Regel nach unten. Die bisherigen Lösungsansätze auf multilateraler Ebene zur Verbesserung der Situation auf den Weltagrarmärkten haben bisher kaum Früchte getragen. Dies gilt sowohl mit Bezug auf die Bemühungen, die Landwirtschaft vermehrt den allgemeinen GATT-Regeln zu unterstellen, als auch hinsichtlich der internationalen Rohstoffpolitik. Von dieser schlechten Verfassung der Weltagrarmärkte werden die verschiedenen Ländergruppen in unterschiedlichem Ausmass betroffen; am nachteiligsten wirkt sie sich jedoch für die auf die Agrarexporte essentiell angewiesenen Staaten und namentlich die Entwicklungsländer aus.

\subsection{Nationale Massnahmen}

Grundsätzlich gelten unsere Schutzmassnahmen an der Grenze auch gegenüber den Drittweltstaaten; in der Regel wird nicht nach Herkunftsländern differenziert. Das agrarhandelspolitische Instrumentarium ist bekanntlich recht komplex und vielfältig. So stehen bei den zahlreichen preislichen Grenzmassnahmen neben Zöllen, Zollzuschlägen und verschiedenen Importabgaben vor allem die Preiszuschläge auf Käse, Speisefetten und -ölen sowie Futtermitteln im Vordergrund. Ebenso reichhaltig ist das Arsenal von quantitativ wirksamen Abwehrmassnahmen. Es erstreckt sich von der relativ liberalen Übernahmepflicht, wo Importbewilligungen wie etwa bei den Eiern und Schaffleisch nur nach Massgabe einer Leistung zugunsten der Inlandsproduktion erteilt werden, über das sogenannte 
Dreiphasensystem bei Früchten und Gemüsen und die Einfuhrkontingentierung bei zentralen Agrarprodukten wie Futtermitteln, Fleisch und Fasswein, bis zu Importmonopolen (Butter, Alkohol) und zu Einfuhrverboten bei einigen wenigen Erzeugnissen.

Die gewichtigste Ausnahme von der Regel der einheitlichen Anwendung der Einfuhrmassnahmen gegenüber den verschiedenen Staatengruppen stellen die sogenannten Zollpräferenzen dar. Darunter werden spezielle, reduzierte Zollansätze verstanden, die Freihandelspartnern und der Dritten Welt gewährt werden. Das Zollpräferenzschema gegenüber den Entwicklungsländern zeichnet sich durch vier Eigenschaften aus:

- Es ist autonomer Natur, das heisst es beruht auf keiner vertraglichen Basis.

- Es ist grundsätzlich einseitig, das heisst von den begünstigten Staaten wird keine entsprechende Gegenleistung enwartet.

- Es ist überdies zeitlich beschränkt, in der Schweiz beispielsweise auf zehn Jahre, bis 1992.

- Ferner ermöglicht im allgemeinen eine Schutzklausel in Ausnahmefällen eine (temporäre) Rücknahme der Zollpräferenzen.

Die Schweiz hat ihr Präferenzschema in zwei Zollabbauschritten anfangs der siebziger Jahre in Kraft gesetzt. Dabei wurden die Zölle auf den meisten Industriewaren völlig oder zumindest zum grossen Teil abgebaut, während nur relativ wenige Landwirtschaftserzeugnisse begünstigt wurden. Weitere Verbesserungen erfolgten 1977 und 1980; im Jahre 1982 schliesslich wurden der Gruppe der ärmsten Entwicklungsländer zusätzliche tarifäre Einfuhrerleichterungen gewährt.

Was die Auswirkungen des schweizerischen Präferenzschemas betrifft, liegen leider keine offiziellen Berechnungen für die Agrarerzeugnisse allein vor. Im Jahr 1979 erreichte die Gesamtheit der tatsächlich präferenziell begünstigten Einfuhren, also inklusive Industrieprodukte, nahezu 1 Milliarde Franken. Dabei gilt es allerdings zu beachten, dass vor allem die wirtschaftlich fortgeschritteneren Drittweltstaaten aus den Präferenzen Nutzen zu ziehen vermochten. So entfielen 1979 70,7 Prozent der unter dem Präferenzenregime getätigten Importe aus den Entwicklungsländern auf zehn Staaten, nämlich Spanien (210 Millionen Franken), Jugoslawien (91 Millionen), Hongkong (72 Millionen), Indien (61 Millionen), Brasilien (59 Millionen), Südkorea (45 Millionen), Israel (37 Millionen), Griechenland (29 Millionen), Mexiko (29 Millionen) und Panama (28 Millionen); Spanien und Griechenland gehörten damals noch zum Kreise der Entwicklungsländer. Neuere Berechnungen sind nicht publiziert worden. Die Marktanteilsverluste der Drittweltstaaten auf dem schweizerischen Agrarmarkt lassen jedoch darauf schliessen, dass die landwirtschaftlichen Zollpräferenzen nur geringe Auswirkungen zeitigen und von den oftmals mit Ausfuhrbeiträgen geförderten Exportanstrengungen anderer Staatengruppen, namentlich der Europäischen Gemeinschaft, überspielt werden. Die beschränkten positiven Effekte der Zollpräferenzen dürtten sich bei den Agrarprodukten allerdings gleichmässiger auf eine grössere Zahl von Entwicklungsländern verteilen als bei den Industriewaren. 
Bei den nichttarifären Schutzmassnahmen an der Grenze besteht für die Entwicklungsländer gegenüber den übrigen Staaten keine Vorzugsbehandlung. So wird beispielsweise bei den verschiedenen agrarpolitisch begründeten Lenkungsabgaben wie Preis- und Zollzuschlägen keine Differenzierung nach Herkunftsländern vorgenommen. Dagegen kommen bei den bilateralen Einfuhrkontingenten selbstverständlich auch die Drittweltstaaten zum Zug. Dies gilt vor allem für roten Fasswein, wo einige Länder des Mittelmeerraums und Südamerikas zum Teil namhafte vertragliche oder autonome Kontingente besitzen. Erwähnung verdient schliesslich auch das Kolumbien im Rahmen der Tokio-Runde des GATT zugesicherte Nelkenkontingent von jährlich 45 Tonnen.

Neben diesen beschränkten staatlichen Massnahmen zur Begünstigung der Agrarimporte aus der Dritten Welt düren die privatrechtlichen Bemühungen, namentlich der Hilfswerke, nicht unerwähnt bleiben. Dabei geht es den Entwicklungsorganisationen nicht um mehr Einfuhren um jeden Preis, sondern um "gerechten" Handel mit den wirtschaftlich unterentwickelten Ländern. So soll namentlich der Absatz von Gütern aus arbeitsintensiver, umweltschonender und kleinbäuerlicher Produktion gefördert werden. In diesem Sinne betreibt die unter anderem von den Hilfswerken getragene Importorganisation OS3 seit längerem über mehrere hundert Verkaufsstellen einen "gerechten" Handel. Dieser entspricht aber beispielsweise beim Kaffee mit 60 Tonnen pro Jahr nur einem Promille der Einfuhren. Die Gesamtagrarimporte von OS3 dürtten einen Wert von einigen wenigen Millionen Franken pro Jahr nicht übersteigen, die Produktepalette ist jedoch recht breit und reicht von verschiedenen Gewürzen über Kaffee und Tee bis zum Honig.

\section{Mehr Agrarimporte aus Entwicklungsländern - Möglichkeiten und Grenzen}

Angesichts der geschilderten Ausgangslage - eher mässiger und rückläufiger Marktanteil der Entwicklungsländer auf dem schweizerischen Agrarmarkt, nur beschränkte Förderung der Exporte landwirtschaftlicher Produkte aus der Dritten Welt - drängt sich die Frage geradezu auf, ob die Zutrittsmöglichkeiten der Entwicklungsländer nicht nachhaltiger verbessert werden solten. Anhand einiger Beurteilungskriterien wird nachfolgend versucht, die komplexe Fragestellung zu beantworten und Möglichkeiten und Grenzen vermehrter Agrarimporte aus den Drittweltstaaten aufzuzeigen.

\subsection{Entwicklungspolitische Überlegungen}

Die Frage der positiven oder negativen Effekte einer Exportförderung in den wirtschaftlich unterentwickelten Ländern auf ihren Entwicklungsprozess ist sowohl in der Theorie als auch in der Praxis umstritten. Dies gilt besonders für die Agrarprodukte, wo eine zu starke Exportorientierung des Landwirtschaftssektors die Versorgung der eigenen Bevölkerung mit Grundnahrungsmitteln gefährden 
kann. Ob die Produktion für den Inlandsmarkt und die Ausfuhr wirklich in einem Konkurrenzverhältnis zueinander stehen, hängt von verschiedenen Faktoren ab, so der sozio-ökonomischen Struktur des Landes, der Bevölkerungsdichte, den natürlichen Ressourcen usw. Somit ergibt sich praktisch für jedes Land eine andere Beurteilung; Brasilien ist mit Mali ebensowenig vergleichbar wie Algerien mit Singapur.

In der neueren Theorie dominieren die handelskritischen Ansätze. So hatten beispielsweise bereits in den sechziger Jahren die Dualismus-Theorie oder Myrdals Modell der zirkularen kumulativen Verursachung auf die gesellschaftspolitisch negativen Auswirkungen einer übereilten Integration der Drittweltstaaten in den Weltmarkt hingewiesen. Während der erste Ansatz die Entwicklungshemmnisse betont, die durch das unverbundene Nebeneinander des Subsistenzsektors und eines marktwirtschaftlichen Sektors als ökonomische Enklave entstehen, macht Myrdal auf die räumlichen Konzentrationsprozesse aufmerksam, die reiche Regionen immer reicher und arme Regionen immer ärmer machen. In den siebziger Jahren haben zwei Strategieansätze an Bedeutung gewonnen, nämlich das Konzept der "Befriedigung der Grundbedürfnisse", und das Abkoppelungsoder Dissoziationsmodell. Danach soll die fortwährende Vertiefung der dualistischen Struktur von Wirtschaft und Gesellschaft aufgehalten und sozusagen eine Entwicklung von unten in Gang gesetzt werden, um die räumliche und sektorale Polarisation in den Drittweltstaaten zu überwinden. Das Schwergewicht wird vor allem auf die Entwicklung des Primärsektors gelegt, wobei seit einigen Jahren auch vermehrt die Notwendigkeit der Erhaltung des ökologischen Gleichgewichts (kein Raubbau!) betont wird.

Dieser eher kritischen Beurteilung aus theoretischer Sicht sind jedoch einige praktische Überlegungen gegenüberzustellen. So ist weiter oben bereits darauf hingewiesen worden, dass die Agrarexporte in vielen Entwicklungsländern eine wichtige Devisenquelle bilden. Bei einem Schuldenberg von heute über 1.000 Milliarden Dollar sind die meisten Drittweltstaaten mehr denn je auf (Agrar-) Ausfuhren angewiesen, damit sie ihrem Schulden- und Zinsendienst einigermassen nachzukommen vermögen. Zudem müssen die Exporteinnahmen eines Landes langfristig in einer vertretbaren Relation zu den Einfuhrbedürnnissen im weitesten Sinne stehen. Im übrigen können die Exporte durchaus positive beschäftigungs- und einkommenswirksame Produktionsimpulse vermitteln, die ihrerseits der Binnennachfrage zugute kommen, insbesondere wenn mit den Ausfuhren eine arbeitsintensivere und ökologisch nicht schädliche Produktion stimuliert wird.

Im allgemeinen dürfte für die Drittweltstaaten eine selektive Kooperation mit der übrigen Welt, verbunden mit einem Abbau der ungleichgewichtigen Entwicklung und der Durchführung von echten Agrarreformen, wohl den erfolgversprechendsten Weg darstellen. Eine massvolle Förderung der Agrarexporte ist somit für viele Länder eine sinnvolle, ja notwendige, keinesfalls aber hinreichende Bedingung für die eigene Entwicklung. Die bisherige Erfahrung hat jedenfalls gezeigt, dass sich Exporterfolge und eine genügende Eigenversorgung nicht gegenseitig ausschliessen müssen; einzelne Drittweltstaaten haben in beiden Bereichen positive Resultate erzielt, andere jedoch auf beiden Ebenen versagt. 


\subsection{Beurteilung aus der Sicht der schweizerischen Agrarpolitik}

Aus agrarpolitischer Sicht ist eine höhere Importquote unerwünscht, da dadurch Produktionsmöglichkeiten und damit auch bäuerliche Einkommen ins Ausland verlagert würden. Dies gilt umso mehr, als die Schweiz im internationalen Vergleich mit etwa 65 Prozent einen relativ niedrigen Brutto-Selbstversorgungsgrad aufweist; die entsprechende Kennziffer liegt beispielsweise in der EG (Zehnergemeinschaft) mit 114 Prozent (1985) und Österreich mit 108 Prozent (1983/84) deutlich höher. Allfällige Mehreinfuhren aus den Entwicklungsländern müssten daher grundsätzlich zu Lasten der übrigen Lieferanten erfolgen. Auf den ersten Blick mag eine solche Importumlagerung als agrarpolitisch irrelevant erscheinen, bei näherer Betrachtung ergeben sich jedoch dennoch gewisse Rückwirkungen auf unsere Landwirtschaft.

Mit Blick auf die Versorgungssicherung, eines der Hauptziele wenn nicht gar das wichtigste Ziel der schweizerischen Agrarpolitik, ist davon auszugehen, dass wir etwa 60 Prozent unserer Agarimporte aus der EG (Zwölfergemeinschaft) beziehen. Bei einigen Produkten (Beispiel Zucker mit über 95 Prozent) ist die EG sogar praktisch Alleinlieferant geworden. Diese Abhängigkeit ist, wie beispielsweise die Schwierigkeiten mit der Zuckerbeschaffung auf dem EG-Markt Mitte der siebziger Jahre gezeigt haben, nicht unbedenklich. Eine etwas bessere Diversifizierung unserer Importstruktur, das heisst vermehrte Bezüge aus Nicht-EG-Staaten und damit auch aus den Entwicklungsländern, würde unsere Versorgungssicherheit tendenziell erhöhen. Stärkere Verschiebungen zwischen den einzelnen Bezugsländern könnten die "Verlierer" allerdings zu Reaktionen veranlassen. So ist nicht ganz auszuschliessen, dass die hauptbetroffene EG, mit der wir handelsvertraglich und -politisch eng verflochten sind, unsere Exporte nach der Zwölfergemeinschaft behindern würde. Da etwa 50 Prozent der Agrarausfuhren und 90 Prozent unserer Käseexporte dorthin gehen, steht einiges auf dem Spiel. Auf der anderen Seite würde es der EG schlecht anstehen, unsere Ausfuhren zu erschweren, nur weil wir die Exporte aus den Entwicklungsländern begünstigen würden, zumal auch die EG verschiedene Drittweltstaaten bereits seit Jahren handelspolitisch bevorzugt (zum Beispiel das Lomé-Abkommen, das u. a. Bezugsverpflichtungen über 1,3 Millionen Tonnen Zucker/Jahr vorsieht).

Schliesslich könnte eine teilweise Verlagerung unserer Agrareinfuhren von der Europäischen Gemeinschaft zu den Entwicklungsländern je nach Produktekategorie eine Verteuerung des Importpreisniveaus zur Folge haben; im Mittel der Jahre 1983/85 kostete uns eine "Agrartonne" aus der EG, unter anderem dank deren Exportsubventionen, geringeren Transportkosten, etc., nur 1.530 Franken, aus der Dritten Welt jedoch 2.400 Franken. Aus der Sicht der schweizerischen Landwirtschaft wäre eine gewisse Verteuerung der Importprodukte in der Regel natürlich erwünscht, da dadurch der Konkurrenzdruck des Auslands abgeschwächt würde. Dort, wo Preisausgleichsmassnahmen an der Grenze möglich sind, müssten allerdings Mindereinnahmen in Kauf genommen werden. Im übrigen wäre eine massvolle Verteuerung der eingeführten Agrarprodukte für den Konsumenten durchaus zumutbar, da die Aufwendungen für Nahrungsmittel in der 
Schweiz relativ niedrig sind und bezogen auf die Haushaltsausgaben (inkl. Versicherungen, Steuern und Gebühren) nur noch 16 bis 18 Prozent ausmachen.

\subsection{Wirtschafts- und ordnungspolitische Überlegungen}

Die schweizerische Wirtschaftspolitik beruht bekanntlich auf dem Prinzip der sozialen Marktwirtschaft, und die Handelspolitik unterliegt weitgehend den Spielregeln des Freihandels. Als stark mit der Weltwirtschaft verflochtenes Land - bald wird jeder zweite Franken im Ausland verdient - ist die Schweiz auf eine möglichst offene, multilaterale Welthandelsordnung angewiesen. Etwas anders verhält es sich dagegen mit der Landwirtschaft. In Anbetracht seiner spezifischen Verhältnisse, beispielsweise der staats- und gesellschaftspolitischen Sonderstellung, ist die Schutzbedürttigkeit des Agrarsektors in der Schweiz wie auch anderswo recht gross. So wird der Landwirtschaftshandel mehr nach interventionistischen als nach liberalen Grundsätzen abgewickelt.

Trotzdem sollte die marktwirtschaftliche Grundphilosophie unserer Wirtschafts- und Handelspolitik auch im Landwirtschaftssektor dort, wo es möglich ist, stärker zum Tragen kommen. Dies gilt beispielsweise mit Bezug auf die Herkunft unserer Agrarimporte. So sollten Lieferländer mit komparativen Kostenvorteilen gegenüber solchen, die ihre Exporte mit Ausfuhrsubventionen künstlich verbilligen, stärker als bisher berücksichtigt werden. Bei verschiedenen Landwirtschaftserzeugnissen besitzen die Entwicklungsländer kosten- und ertragsmässig längere Spiesse, die für Mehrbezüge von Agrarprodukten aus den Drittweltstaaten sprechen (Beispiel Zucker). Durch eine solche teilweise Umverlagerung ihrer Landwirtschaftseinfuhren von der EG zu den Entwicklungsländern könnte die Schweiz aus ordnungspolitischer Sicht einen positiven Beitrag zu einer verbesserten internationalen Arbeitsteilung und räumlichen Allokation der Produktionsfaktoren leisten. Auf der anderen Seite erfordert eine zukunftsbezogene Betrachtungsweise eine vermehrte Produktion und Ausfuhr verarbeiteter Erzeugnisse mit höherer Einkommenselastizität, denn nur so haben die Entwicklungsländer auf längere Sicht eine Chance, durch einen grösseren Anteil an Wachstumsbranchen aus dem Teufelskreis der Unterentwicklung auszubrechen.

Etwas anders präsentiert sich die Situation aus rein binnenwirtschaftlicher Sicht. Ordnungspolitische Überlegungen sprechen für eine möglichst grosse Dispositionsfreiheit des Handels, d.h. gegen Bezugsverpflichtungen aus bestimmten Staaten oder Ländergruppen. Eine staatliche verordnete Umleitung der Handelsströme auf die Entwicklungsländer wäre mit unserem liberalen Wirtschaftssystem nicht ohne weiteres vereinbar, zumal die Importeure bezüglich Lieferland im allgemeinen frei sind, und zwar auch bei den Agrarprodukten. Von diesem bewährten Grundsatz sollte nur dann abgewichen werden, wenn per Saldo die Argumente für Mehrimporte aus den Drittweltstaaten eindeutig überwiegen. Besser wäre jedoch, wenn Staaten mit Standortnachteilen auf eine übermässige Agrarexportsubventionierung verzichten würden, so dass von selbst die wettbewerbskräftigsten Länder vermehrt zum Zuge kämen. 


\subsection{Lösungsansätze}

Zur Verbesserung der Agrarexportmöglichkeiten der Entwicklungsländer nach der Schweiz (und in die übrigen Industrieländer), ohne jedoch gleichzeitig die Ernährungsbasis der Drittweltstaaten zu gefährden, ist der Hebel auf verschiedenen Ebenen anzusetzen. Zunächst in den Lieferländern selbst, dann aber auch bei den multilateralen Rahmenbedingungen für den Weltagrarhandel und schliesslich in den einzelnen Bezugsstaaten.

Ohne eine Stärkung der Landwirtschaft in den Drittweltstaaten dürften die übrigen Massnahmen weitgehend ins Leere stossen. Die meisten Entwicklungsländer haben den Industriesektor gegenüber dem Agrarsektor einseitig begünstigt, und verschiedene Drittweltstaaten erschweren sogar ihre Ausfuhren von Landwirtschaftsprodukten durch zum Teil hohe Exportsteuern. Der historischen Erfahrung, wonach einem dynamischen Agrarsektor zur Förderung der Gesamtwirtschaft eine ausschlaggebende Rolle zukommt, ist bisher jedenfalls meistens zu wenig Rechnung getragen worden. Eine Aufwertung der Landwirtschaftspolitik in den Entwicklungsländern muss daher als conditio sine qua non für eine Verbesserung der sozio-ökonomischen Bedingungen in den Drittweltstaaten bezeichnet werden. Durch günstigere Voraussetzungen, namentlich lohnende Produktepreise und eine vermehrte Förderung der ländlichen Räume ganz allgemein, sind die notwendigen Anreize zu schaffen, damit die Bauern über ihre eigenen Bedürfnisse hinaus überschüssige Nahrungsmittel zur Versorgung der lokalen Märkte und für den Export produzieren.

Die Eigenanstrengungen der Entwicklungsländer müssen jedoch von verbesserten Rahmenbedingungen für den Weltagrarhandel begleitet sein. Durch eine stetigere, transparentere und verhältnismässigere Agrarpolitik in den Industriestaaten sind die strukturellen Überschüsse abzubauen, übermässige Exportsubventionen zu vermindern und die Weltmarktpreise für Landwirtschaftsprodukte auf einem höheren Niveau zu stabilisieren. Somit könnte der Agrarhandel wieder vermehrt die echten Kosten- und Ertragsverhältnisse widerspiegeln, indem stärker als heute die standortbegünstigten Produzentenländer und namentlich die Drittweltstaaten zum Zuge kämen. Eine der wichtigsten, aber auch der schwierigsten Aufgaben der Uruguay-Runde des GATT wird darin bestehen, solchen marktwirtschaftlichen Grundsätzen im Agrarhandel vermehrt zum Durchbruch zu verhelfen, gleichzeitig aber den legitimen Schutzbedürfnissen der Landwirtschaft in den Importstaaten Rechnung zu tragen. Die Sonderstellung des Agrarsektors darf aber den Industriestaaten mit komparativen Standortnachteilen nicht als Vorwand zu einer vollständigen Abschottung ihrer Landwirtschaft vom Weltmarkt und von der übrigen Wirtschaft dienen.

Schliesslich kann und soll auch die Schweizihren Beitrag zur Unterstützung der Agrarexportbemühungen der Entwicklungsländer leisten. Zunächst und vor allem auf multilateraler Ebene, indem sie sich getreu ihrer freiheitlichen Wirtschafts- und Handelspolitik soweit möglich für eine vermehrte Beachtung marktwirtschaftlicher Spielregeln auch im Landwirtschaftshandel einsetzt. Im bilateralen Bereich sind Agrarimporte aus Drittweltstaaten, dort wo es entwicklungspolitisch sinnvoll und 
agrarpolitisch tragbar ist, gezielt zu fördern (zum Beispiel Zucker), sei es durch einen Ausbau des Zollpräferenzensystems oder durch andere geeignete Vorkehren. Wichtig wäre auch ein Ausbau konkreter handelsfördernder Massnahmen wie die Mithilfe bei der Vermarktung von Agrarerzeugnissen aus den Entwicklungsländern in der Schweiz, beispielsweise durch die Finanzierung der Teilnahme von Drittweltstaaten an Messen und Ausstellungen oder die Förderung der Qualität und Marktnähe der Produkte.

\section{Literaturverzeichnis}

Banque mondiale, La politique du commerce et des prix dans le domaine de l'agriculture mondiale, in: Rapport sur le développement dans le monde 1986, Washington 1986.

A. Basler, Neuere entwicklungspolitische Theorieansätze und ihre Bewertung im Hinblick auf Nahrungsversorgung und Weltagrarhandel, in: Agrarwirtschaft, August 1979, Heft 8.

M. Bohnet, Die Entwicklungstheorien - ein Überblick, in: Das Nord-Süd-Problem, Konflikte zwischen Industrie- und Entwicklungsländern, München 1971.

Botschaft vom 25.2.1981 über handels- und rohstoffpolitische Massnahmen im Bereich der Entwicklungszusammenarbeit, BBI 1981 II I.

Botschaft vom 19.2.1986 über die Weiterführung der Finanzierung von wirtschafts- und handelspolitischen Massnahmen im Rahmen der Entwicklungszusammenarbeit, BBI 1986 I 1289.

Botschaft vom 2.3.1987 über die Weiterführung der technischen Zusammenarbeit und der Finanzhilfe zugunsten von Entwicklungsländern, BBI 1987 II I.

Bundesamt für Aussenwirtschaft, Commerce extérieur agricole suisse (erscheint jährlich).

Eidg. Oberzolldirektion, Aussenhandelsstatistik, Band I und II, diverse Jahrgänge.

FAO, Rapport et perspectives sur les produits 1985-86, Rome 1986.

FAO, Trade Yearbook 1985, Rome,1986.

R. Horber, Die schweizerische Aussenhandelspolitik mit den Entwicklungsländern - Fakten und Probleme, in: Wirtschaftsförderung, Dokumentation zur Wirtschaftskunde Nr. 105, Dezember 1986.

Neunter Entwickungsbericht der Weltbank, Zusammenfassung der NZZ vom 8.7.1986, "Freihandel als Chance für Entwicklungsländer".

OCDE, Echanges agricoles avec les pays en développement, Paris 1984.

Schweizer Hilfswerke, Für gerechten Handel mit der Dritten Welt, 1986.

Sechster Landwirtschaftsbericht des Bundesrates, Bern 1984. 